\title{
On the structure of solid lipid nanoparticles
}

DOI:

10.1002/smll.201903156

\section{Document Version}

Accepted author manuscript

Link to publication record in Manchester Research Explorer

\section{Citation for published version (APA):}

Pink, D. L., Loruthai , O., Ziolek, R. M., Wasutrasawat, P., Terry, A. E., Lawrence, J., \& Lorenz, C. D. (2019). On the structure of solid lipid nanoparticles. Small, 15(45), [1903156]. https://doi.org/10.1002/smll.201903156

\section{Published in:}

Small

\section{Citing this paper}

Please note that where the full-text provided on Manchester Research Explorer is the Author Accepted Manuscript or Proof version this may differ from the final Published version. If citing, it is advised that you check and use the publisher's definitive version.

\section{General rights}

Copyright and moral rights for the publications made accessible in the Research Explorer are retained by the authors and/or other copyright owners and it is a condition of accessing publications that users recognise and abide by the legal requirements associated with these rights.

\section{Takedown policy}

If you believe that this document breaches copyright please refer to the University of Manchester's Takedown Procedures [http://man.ac.uk/04Y6Bo] or contact uml.scholarlycommunications@manchester.ac.uk providing relevant details, so we can investigate your claim.

\section{OPEN ACCESS}




\title{
On the structure of solid lipid nanoparticles
}

\author{
Demi L. Pink ${ }^{1}$, Orathai Loruthai ${ }^{2}$, Robert M. Ziolek ${ }^{1}$, Prawarisa Wasutrasawat ${ }^{3}$, Ann E. \\ Terry $^{4}$, M. Jayne Lawrence ${ }^{5}$, and Christian D. Lorenz ${ }^{1}$ \\ ${ }^{1}$ Department of Physics, King's College London, London, WC2R 2LS, UK \\ ${ }^{2}$ Pharmaceutical Biophysics Group, Institute of Pharmaceutical Science, King's College \\ London, London, SW1 9NH, UK \\ ${ }^{3}$ Department of Pharmaceutics and Industrial Pharmacy, Faculty of Pharmaceutical \\ Sciences, Chulalongkorn University, Bangkok, 10330, Thailand \\ ${ }^{4}$ CoSAXS beamline, MAX IV Laboratory, Lund University, P.O. Box 118, SE-221 00 Lund, \\ Sweden \\ ${ }^{5}$ Division of Pharmacy and Optometry, School of Health Sciences, Faculty of Biology, \\ Medicine and Health, University of Manchester, Stopford Building, Oxford Road, \\ Manchester, UK
}

September 6, 2019

\begin{abstract}
Solid lipid nanoparticles (SLNs) have a crystalline lipid core which is stabilised by interfacial surfactants. SLNs are considered favorable candidates for drug delivery vehicles since their ability to store and release organic molecules can be tailored through the identity of the lipids and surfactants used. When stored, polymorphic transitions in the core of drug-loaded SLNs lead to the premature release of drug molecules. Significant experimental studies have been conducted with the aim of investigating the physico-chemical properties of SLNs, however, no molecular scale investigations have been reported on the behaviors that drive SLN formation and their polymorphic transitions. We have therefore used a combination of small angle neutron scattering (SANS) and all-atom molecular dynamics simulations (MS) to yield a detailed atomistic description of the internal structure of an SLN comprising of triglyceride, tripalmitin, and the nonionic surfactant, Brij $\mathrm{O} 10\left(\mathrm{C}_{18: 1} \mathrm{E}_{10}\right)$. We uncover the molecular scale mechanisms by which the surfactants stabilise the crystalline structure of the SLN lipid core. By comparing these results to simulated liquid and solid aggregates of tripalmitin lipids, we demonstrate how the morphology of the lipids vary between these systems providing further insight into the mechanisms that control drug encapsulation and release from SLNs.
\end{abstract}

\section{Introduction}

Solid lipid nanoparticles (SLNs) are colloidal drug delivery systems, ranging in size from 10 - $1000 \mathrm{~nm}$. They are comprised of solid lipids stabilized by a layer of surface active (surfactant) molecules. Since their discovery in the early 1990 s, SLNs ${ }^{[1],[2],[3]}$ have emerged as a promising delivery system for small therapeutic molecules, particularly lipophilic drug molecules ${ }^{[4],[5]}$. SLNs have exhibited considerable potential for the targeted delivery of anticancer agents ${ }^{[6]}$. The strong interest in SLNs as drug delivery vehicles partially arises from their low toxicity ${ }^{[7],[8]}$ and green synthesis (no organic solvents are required in their preparation) ${ }^{[7]}$. Probably their most significant advantage, however, is that they can be created using lipids that remain solid at room temperature, and therefore encapsulate the therapeutic agent within their solidified lipid matrix $[9],[10],[11],[12],[13]$. The SLN then become fluid at body temperature and release the entrapped therapeutic 
agents ${ }^{[8]}$. The choice of the material(s) comprising the lipid matrix component can be selected to control the melting temperature of the SLNs. It has been established that, depending upon the solubility of the therapeutic agent, the method of SLN preparation can also influence the therapeutic agent's location within the SLN. The location of the drug in the SLN can in turn affect its release ${ }^{[14]}$. For example, if the entrapped therapeutic agent is located predominately at the surface of the SLN, it is released more rapidly than if it were located in the lipid core ${ }^{[7]}$. The rate of drug release can therefore be controlled by the choice of lipids and the solubility of the encapsulated therapeutic agent.

One challenge facing the commercial exploitation of SLNs as drug delivery vehicles is their instability $[10],[15],[16]$. This instability is due at least in part to the tendency of the lipid core to first crystallize in the least ordered, metastable $\alpha$-form, but then slowly transform into the more ordered, more stable $\beta$-form $[17],[18]$. During the polymorphic transition from the $\alpha$-form to the $\beta$-form, the lipid molecules rearrange, causing a change in the morphology of the SLN from spherical ( $\alpha$-form) to elongated, platelet-shaped $(\beta$ form) SLNs $[10],[15],[16],[19]$. However, the transition towards the more ordered $\beta$ lattice removes defects, in which the lipophilic therapeutic agents generally sit, which can cause the expulsion of the therapeutic agent from the SLN during storage or administration ${ }^{[7]}$. Another consequence of this transition is that the surface-to-volume ratio of the SLN increases, generating new surfaces, which may result in attractive hydrophobic interactions between the individual SLN particles, inducing instability through particulate aggregation and gelation ${ }^{[10],[19]}$. The surfactant molecules that comprise the stabilizing monolayer serve to reduce this aggregation by shielding the hydrophobic lipid chains upon the transition from the $\alpha$-form to the $\beta$-form.

Although there has been significant experimental research dedicated to determining the physico-chemical properties (including stability) of SLNs, there is currently little understanding of the detailed molecular structure of these nanoparticles. Furthermore, while molecular dynamics (MD) simulations have been used to investigate the molecular scale structure and nucleation processes of bulk crystalline lipids [20], [21],[22],[23],[24],[25],[26],[27], there are few simulation studies of lipid nanoparticles. The few that have been conducted have studied liquid, not solid, nanoparticles. In one study, coarse-grained MD simulations were used to investigate the self-assembly of the triglyceride, tripalmitin and the nonionic surfactant, Tween 20, into surfactant coatedliquid lipid nanoparticles. Subsequently, docking simulations were used to predict the loading of therapeutic agents into the core of a SLN ${ }^{28]}$. Significantly, there has been no work published on the simulation of SLNs at the atomistic level as a means of determining the detailed molecular structure of these systems.

In our previous work, we have used various experimental methods to investigate SLN formed from tripalmitin, a lipid with three polar ester groups connected to three saturated hydrophobic chains (see Fig. 1). We characterized the capability of SLNs formed of tripalmitin and the nonionic $\mathrm{C}_{18: 1} \mathrm{E}_{10}$ surfactant to load the drug testosterone propionate ${ }^{[29]}$. Additionally, SLNs consisting of tripalmitin and a range of surfactants have been shown to exist in the $\alpha$-form polymorphic state, and vary in size from $10-230 \mathrm{~nm}$ in diameter by us ${ }^{[29]}$ and others ${ }^{[16]}$.

In this manuscript, we present the first description of molecular-scale mechanisms that are important with regards to the formation and morphology of tripalmitin SLN using all-atom MD simulations and small angle neutron scattering (SANS) experiments. We have used MD simulations to determine the difference in the structure and conformation of the lipids in solid lipid and liquid lipid aggregates. We then demonstrate how the $\mathrm{C}_{18: 1} \mathrm{E}_{10}$ surfactant affects the conformation of the tripalmitin molecules within the solid lipid core, as well as the overall morphology of the nanoparticle. 


\section{Methods}

\section{Experimental}

\section{Materials}

All materials obtained commercially were used as received. Tripalmitin (purity 99\% (99hTPN) and 85\% (85hTPN)) and $\mathrm{C}_{18: 1} \mathrm{E}_{10}$ (polyoxyethylene-10-oleyl ether) were supplied by Sigma Chemical Company Ltd. (Poole, Dorset). $\mathrm{D}_{2} \mathrm{O}$ (> 99.9\%) was obtained from Aldrich Chemical Company Ltd. (Gillingham, Dorset). Triple-distilled water obtained from a well- seasoned all-glass still was used throughout.

\section{Preparation and Preliminary Measurements of Solid Lipid Nanoparticles}

Two methods were used for the preparation of the ternary solid lipid nanoparticles (SLNs), namely the phase inversion method in which the required weights of $85 \mathrm{~h}$ TPN, $\mathrm{C}_{18: 1} \mathrm{E}_{10}$ and $\mathrm{H}_{2} \mathrm{O}$ were mixed, the resultant mixture heated to $343-348 \mathrm{~K}$ for 10 minutes followed by cooling to ambient with vigorous and constant stirring and the sonication method in which probe sonication in place of heating the sample until the solution homogeneously mixed and the mixtures stirred at room temperature. Samples which were denoted as being SLNs (i.e. one phase, isotropic and transparent, opalescent or cloud/milky) and stable for at least a month. It is of note for the present studies that samples prepared using the same oil:surfactant weight ratio but different surfactant concentrations, exhibited the same apparent hydrodynamic size (as determined by dynamic light scattering) ${ }^{[29]}$. The only exceptions being the SLNs prepared with high ratios (i.e. 0.8:1 and above) of oil:surfactant where very slight increases in the apparent hydrodynamic size measured by dynamic light scattering was seen (as shown in the SI). The phase inversion method was preferred over the sonication method due to its ease and speed of preparation, and the avoidance of contamination of the metal precipitation that may occur during sonication. As it was not possible to directly prepare SLNs at the $\mathrm{C}_{18: 1} \mathrm{E}_{10}$ concentrations selected for the small angle neutron scattering (SANS) studies, the SLNs were instead made at higher $\mathrm{C}_{18: 1} \mathrm{E}_{10}$ concentrations and diluted to the required surfactant concentration, namely $2 \% \mathrm{w} / \mathrm{w}$. Preliminary SANS experiments showed that the samples prepared using the same oil:surfactant ratio but using different concentrations of surfactant yielded the same size particles and that SLNs of less than $5 \% \mathrm{w} / \mathrm{w}$ showed no evidence of inter-particulate interactions, whereas higher concetrations exhibited these interactions ${ }^{[30]}$.Furthermore, the diluted samples were stable for periods of over 1 month.

\section{Small angle neutron scattering}

Small angle neutron scattering (SANS) experiments were performed on the SANS2d small-angle diffractometer at the ISIS Pulsed Neutron Source (STFC Rutherford-Appleton Laboratory, Didcot, Oxford) using a 12 $\mathrm{mm}$ diameter circular beam and a sample-to-detector distance of $8 \mathrm{~m}$. Neutrons of wavelengths in the range 2-14 $\AA$ were used, giving a scattering vector $Q$, where:

$$
Q=(4 \pi / \lambda) \sin (\theta / 2)
$$

in the range $0.0045-0.8 \AA$. The neutron scattering pattern was recorded on ${ }^{3} \mathrm{He}_{-} \mathrm{CF}_{4}$ filled ORDELA detectors (dimensions of $96.5 \times 96.5 \mathrm{~cm}^{2}, 5 \mathrm{~mm}$ resolution). The transmission and scattering runs of the sample and the $\mathrm{D}_{2} \mathrm{O}$ solvent were measured simultaneously using $2 \mathrm{~mm}$ path length Hellma quartz cuvettes at $25 \pm 0.1{ }^{\circ} \mathrm{C}$. To create a composite SANS pattern, wavelength dependent corrections were performed to allow for the incident spectrum, detector efficiencies and measured sample transmissions. Comparisons with scattering from a partially deuterated polystyrene standard allowed the absolute scattering cross section to be determined, with an approximate error of $\pm 2 \%$.

All fitting procedures were performed using the FISH software ${ }^{[31]}$ and assumed a flat background correction to allow for any mismatch in the incoherent and inelastic scattering between the samples and solvent. Fitted 
background levels were always checked to ensure that they were of a physically reasonable magnitude. The absolute SANS intensity of scattering $\frac{\delta \Sigma}{\delta \Omega}(Q)$ for $\mathrm{N}$ discrete particles per unit volume, in solution is given by:

$$
\frac{\delta \Sigma}{\delta \Omega}(Q)=N \times P(Q) \times S(Q)
$$

where the form factor $P(Q)$ is $\left[|F(Q)|^{2}\right]$, a function that describes how $\frac{\delta \Sigma}{\delta \Omega}$ is modulated by interference effects between radiations scattered by different parts of the same scattering body. In particular, the form factor describes the scattering observable from an isolated body in the absence of interference effects and depends on the particle size and shape. $S(Q)$ is the interparticle structure factor which represents the effect of interference due to neighbouring scattering centres. A range of possible shapes for the SLNs were examined including spherical, ellipsoidal (oblate or prolate), and rods. In the present study, the SANS data of the SLNs in $\mathrm{D}_{2} \mathrm{O}$ were well modelled assuming the presence of monodisperse, core-shell ellipsoids. Preliminary studies explored the existence of interparticulate interactions present, assuming hard-sphere interactions using the Percus-Yevick approximation ${ }^{[30]}$. In the present study at the $\mathrm{C}_{18: 1} \mathrm{E}_{10}$ concentration studied, there was no evidence of any interactions in the systems studied so that there was no need to account for hard sphere interactions ${ }^{[30]}$.

\section{Molecular dynamics simulations}

The structures of the lipid and surfactant molecules were created using Avogadro ${ }^{[32]}$. The CHARMM General Force Field (CGenFF) ${ }^{[33]}$ was used to model the tripalmitin lipid and $\mathrm{C}_{18: 1} \mathrm{E}_{10}$ surfactant molecules. The water molecules were modeled with the CHARMM TIP3P model ${ }^{[34]}$.

The SLN are simulated at the same lipids:surfactant ratio as was used for the SANS experiments (1:2.5). These simulations are computationally expensive due to the large number of solvent molecules, consequently this expense was minimized by self-assembling an initial lipid aggregate. Aggregation numbers based on the SANS experiments (as reported in the Results section) were used to build our simulated systems. Therefore, 229 tripalmitin molecules were placed randomly in a simulation box which was then filled with water. The system was minimized and equilibrated at a pressure of $1 \mathrm{~atm}$ and a temperature of $353 \mathrm{~K}$ for $2 \mathrm{~ns}$. The production simulation ran for $120 \mathrm{~ns}$ using the NPT (Number of atoms, Pressure and Temperature) ensemble. An initial simulation temperature of $353 \mathrm{~K}$ was selected to accelerate lipid aggregation. After $20 \mathrm{~ns}$, simulated annealing was used to cool the system from $353 \mathrm{~K}$ to $310 \mathrm{~K}$ over a period of $50 \mathrm{~ns}$. The temperature was then kept fixed at $310 \mathrm{~K}$ for the remainder of the simulation (50 ns). A temperature of $310 \mathrm{~K}$ was selected as it is slightly below the re-crystallization temperature of $\alpha$-form tripalmitin $(315 \mathrm{~K})$. At the end of the production simulation, an aggregate had formed that will be referred to as the 'Initial Aggregate' (IA).

\begin{tabular}{ccccc}
\hline System & Lipid & Surfactant & Solvent & Total Atoms \\
\hline Solid Aggregate (SA) & $229(10 \% \mathrm{wt})$ & 0 & 92,146 & 311,933 \\
Liquid Aggregate (LA) & $229(10 \% \mathrm{wt})$ & 0 & 92,146 & 311,933 \\
Solid Lipid Nanoparticle (SLN) & $229(6 \% \mathrm{wt})$ & $650(15 \% \mathrm{wt})$ & 135,031 & 521,838 \\
\hline
\end{tabular}

Table 1: Details of the different systems simulated, including the number of lipid, surfactant and solvent molecules present in each simulation.

The IA was used as the basis for the simulation of the three systems that have been investigated in this manuscript. For the liquid aggregate (LA), additional water molecules were added to the IA to obtain the experimentally relevant concentration. The system was minimized and equilibrated as before and a production simulation was run in the NPT ensemble at $353 \mathrm{~K}$ for $120 \mathrm{~ns}$. This resulted in the formation of the LA. To simulate the solid aggregate (SA), the final frame of the LA was extracted and a production run 
was initiated in the NPT ensemble with a temperature of $353 \mathrm{~K}$. After $20 \mathrm{~ns}$ at $353 \mathrm{~K}$, the temperature was decreased over $55 \mathrm{~ns}$ to $310 \mathrm{~K}$ to form the SA and the remainder of the simulation was run at $310 \mathrm{~K}$. The SA was simulated for a total of $290 \mathrm{~ns}$. In order to simulate the solid lipid nanoparticle (SLN), 650 surfactant molecules were placed randomly in solution around the IA. The resulting concentrations of surfactant and lipid molecules in the SLN system were $15 \%$ wt and $6 \% \mathrm{wt}$, respectively. The total number of lipid, surfactant and water molecules in each simulated system is shown in Table 1. The system energy was minimized and equilibrated as above. During the 120 ns production run, the NPT ensemble was used to equilibrate the system at $310 \mathrm{~K}$ and $1 \mathrm{~atm}$. The formation of the SLN is based on experimental protocol of SLN production known as cold homogenization. All simulations were carried out using Gromacs 5.1.2 [35],[36],[37],[38].

\section{Analysis of lipid conformations}

Triglycerides can adopt one of several different conformations depending on their environment. In order to determine the conformations of the tripalmitin molecules, three vectors (shown in Fig. 1) were defined where the vector connects the central carbon of the headgroup (C18) to each of the terminal carbons (C1, C35, C51) as has been done before in the analysis of bulk tripalmitin ${ }^{[20]}$. Calculating the vector angles allowed each lipid to be classified into one of the conformations (trident, propeller, tuning fork and chair) shown in Fig. 2. The angle criteria used to classify a triglyceride molecule is summarized in the SI. It is the same as used in a previous study however this work defines the vectors from the central carbon (C18) as opposed to the first oxygen in each chain ${ }^{[24]}$. Determining the length of each vector also provided us with an understanding of lipid extension, as shown in the SI.

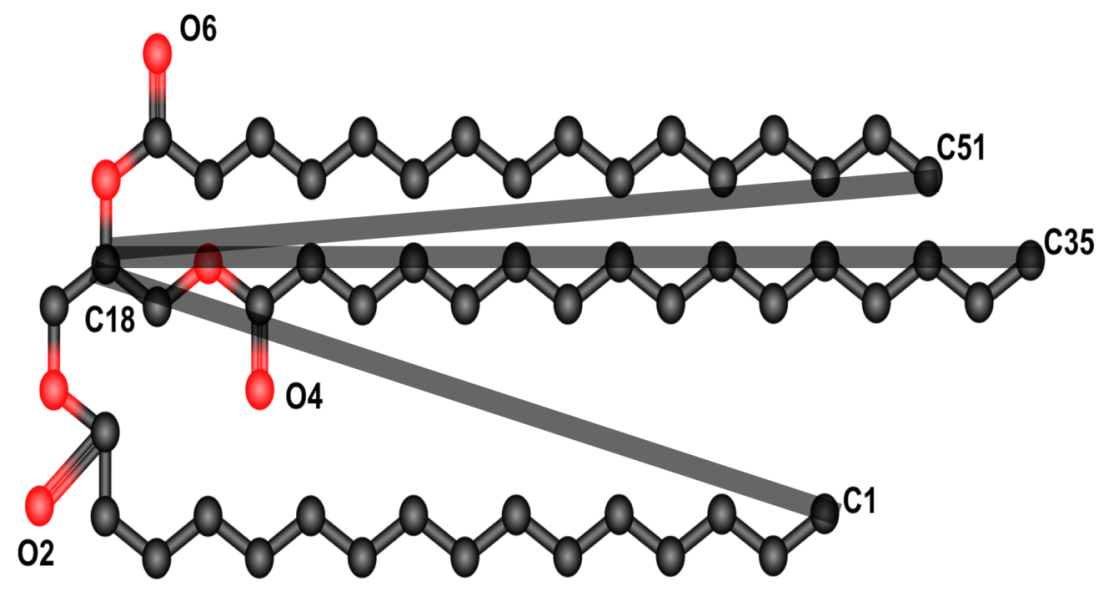

Figure 1: Structure of the tripalmitin lipid molecule with atoms used for analysis labeled.

A detailed picture of nanoparticle morphology emerged from studying how different lipid conformations are distributed throughout the nanoparticle. In this work 'nanoparticle' is used to refer to the entirety of the system in question; this means all lipids in the LA and SA, and both the lipid and surfactant in the SLN. 'Lipid aggregate' refers only to the lipids in each system. Additional description of the analysis of the MD trajectories is provided in the SI. 
a.

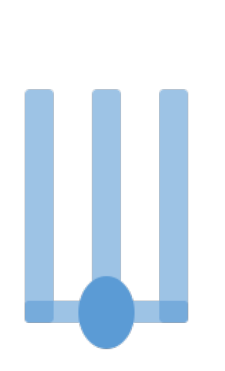

b.

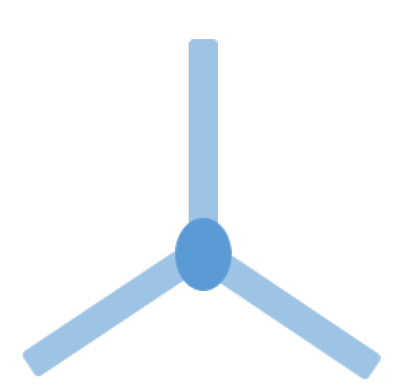

c.

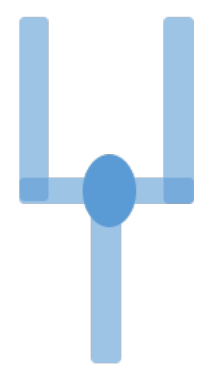

d.

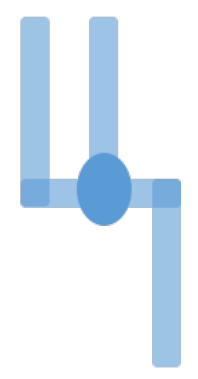

Figure 2: Depictions of the (a) trident, (b) propeller, (c) tuning fork and (d) chair lipid conformations. The position of the central C18 atom is indicated by the blue circle; each blue line represents one of the triglyceride chains.
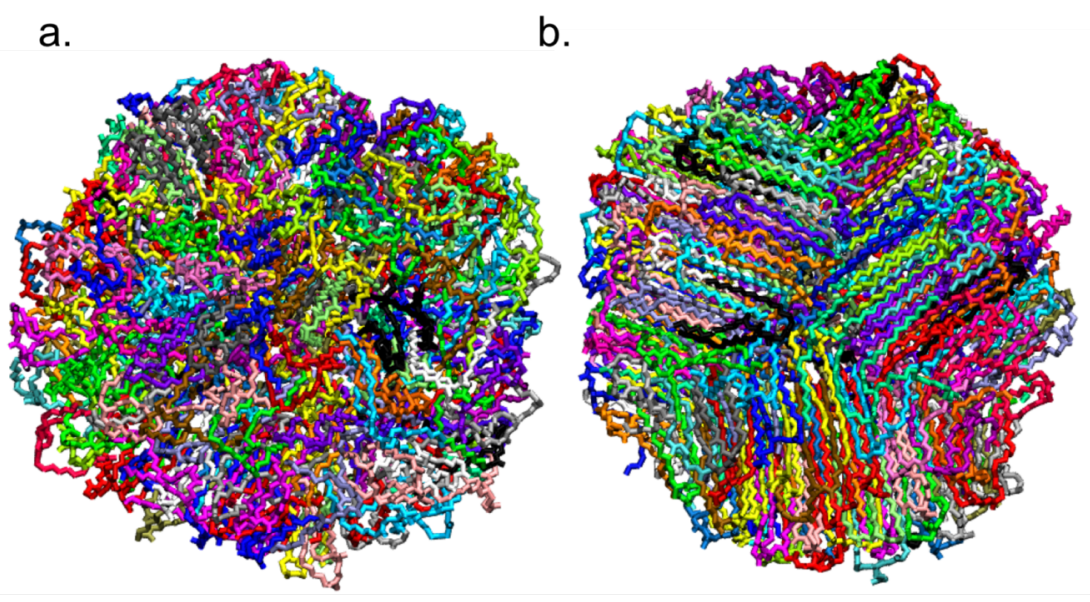

Figure 3: Snapshots of the equilibrated structure of (a) the liquid aggregate (LA) and (b) the solid aggregate (SA). Each lipid molecule is represented in a different color for clarity.

\section{Results}

\section{Liquid Aggregate (LA) and Solid Aggregate (SA)}

Visual inspection of the simulation trajectories using VMD ${ }^{[39]}$ showed the formation of spherical aggregates for the LA and SA. Fig. 3 demonstrates the clear difference in the structure of the SA compared to the LA. In the LA, the lipids form an amorphous nanoparticle. While in the SA, the lipid molecules are significantly more ordered and several facets of crystalline lipid molecules can be observed.

As lipids transition from the liquid phase to a solid phase, their tails extend due to additional van der Waals interactions between them (see SI). The vector lengths for the LA and the SA are reported in Table 2. The increase in lipid chain extension demonstrates an increase in lipid order and a reduction in chain flexibility. This observation is consistent with the transition from liquid lipid molecules to solid lipid molecules. As a result, this work uses the stabilization of the vector lengths (as defined in Fig. 1) to confirm the formation of stable aggregates.

The RDFs of C18 atoms in the triglyceride (see SI) also differentiate between liquid lipid molecules and solid 


\begin{tabular}{cccccc}
\hline & $\mathbf{C 1 8}-\mathbf{C 1}(\mathbf{n m})$ & $\mathbf{C 1 8}-\mathbf{C 5 1}(\mathbf{n m})$ & $\mathbf{C 1 8}-\mathbf{C 3 5}(\mathbf{n m})$ & $R_{g}(\mathbf{n m})$ & Eccentricity \\
\hline LA & $1.58 \pm 0.02$ & $1.53 \pm 0.02$ & $1.58 \pm 0.02$ & $3.43 \pm 0.01$ & 0.07 \\
SA & $1.80 \pm 0.01$ & $1.74 \pm 0.01$ & $1.79 \pm 0.01$ & $3.31 \pm 0.01$ & 0.08 \\
SLN & $1.82 \pm 0.01$ & $1.75 \pm 0.01$ & $1.82 \pm 0.01$ & $5.53 \pm 0.01$ & 0.08 \\
\hline
\end{tabular}

Table 2: Lipid vector lengths, radius of gyration $\left(R_{g}\right)$ and the eccentricities for each system.

\begin{tabular}{ccccccc}
\hline & & Trident & Propeller & Tuning Fork & Chair 1 & Chair 2 \\
\hline Overall (\%) & LA & 78.2 & 6.4 & 6.3 & 4.1 & 5.0 \\
& SA & 82.9 & 5.9 & 4.7 & 3.7 & 2.8 \\
& SLN & 85.5 & 1.9 & 5.8 & 3.6 & 3.2 \\
\hline Distance (nm) & LA & 2.49 & 1.48 & 1.48 & 1.68 & 1.89 \\
& SA & 2.36 & 1.70 & 1.74 & 1.98 & 2.05 \\
& SLN & 2.66 & 2.02 & 1.19 & 1.64 & 1.77 \\
\hline
\end{tabular}

Table 3: Lipid composition of liquid aggregate (LA), solid aggregate (SA) and solid lipid nanoparticle (SLN) throughout the aggregates. Lipids are classified as propeller, trident, tuning fork or chair. The mean minimum distance between each lipid conformation and the COM of the entire lipid nanoparticle is reported for the LA and the SA systems. Note: Chair 1 and Chair 2 refer to the two chair conformation isomers.

lipid molecules. Combined, the RDFs and vector lengths show that the SA and SLN aggregates consist of more ordered crystalline lipids than the LA. The low eccentricity values (see Table 2) confirm that all of the aggregates are approximately spherical.

\section{Distribution of lipid conformations}

As mentioned previously, the triglyceride molecules are characterized by their conformation. Table 3 details the percentage of each conformation found in the LA and the SA. It is clear, although unsurprising, that the trident conformation dominates in both aggregates. The amphiphilic nature of triglycerides means that the trident conformation is the most favorable at the lipid-water interface. In the trident conformation, the lipid molecules can self-assemble in a manner which maximizes the interactions between water and the polar ester groups whilst also minimizing water interactions with the hydrophobic carbon tails. The ester groups have a much larger total solvent accessible surface area $(\mathrm{SASA})\left(\approx 24.4 \mathrm{~nm}^{2}\right.$ for LA; $\approx 27.5 \mathrm{~nm}^{2}$ for SA) than the terminal carbons $\left(\approx 0.85 \mathrm{~nm}^{2}\right.$ for $\mathrm{LA} ; \approx 0.83 \mathrm{~nm}^{2}$ for SA). Therefore, the ester groups are found at the lipid-water interface whilst the lipid tails make up the majority of the nanoparticle's internal volume, effectively shielding themselves from water.

When considering the overall structure of the LA and SA, the location of each lipid within the nanoparticles was determined and its conformation recorded. Fig. 4 shows a symmetric distribution of the trident shaped lipids centered at $2.5 \mathrm{~nm}$ from the nanoparticle COM in the LA and $2.4 \mathrm{~nm}$ for the SA. Other lipid conformations display more skewed distributions, with peaks either side of the trident mean value. The mean minimum distances from the COM of each nanoparticle are shown in Table 3.

The analysis of lipid conformation and spatial distribution suggests a shell of trident lipids at the interface with the aqueous environment. Trident lipids make up the majority of the lipids at the interface but are not present at the center of the aggregate (see Fig. 4). The shell of trident lipids in the SA is formed of several facets of crystalline and ordered lipids whilst the shell of trident lipids in the LA is liquid and disordered. The non-trident lipid conformations primarily occupy the core (as shown by Table 3), with very few present at the surface of the lipid aggregates. The few non-trident lipids at the interface are located at the surface of the crystalline subcells of trident lipids thereby protecting the hydrocarbon tails of the trident lipids that would otherwise be exposed to the aqueous environment.

While the general lipid morphology and distribution is similar in the LA and SA, the trident lipids are 


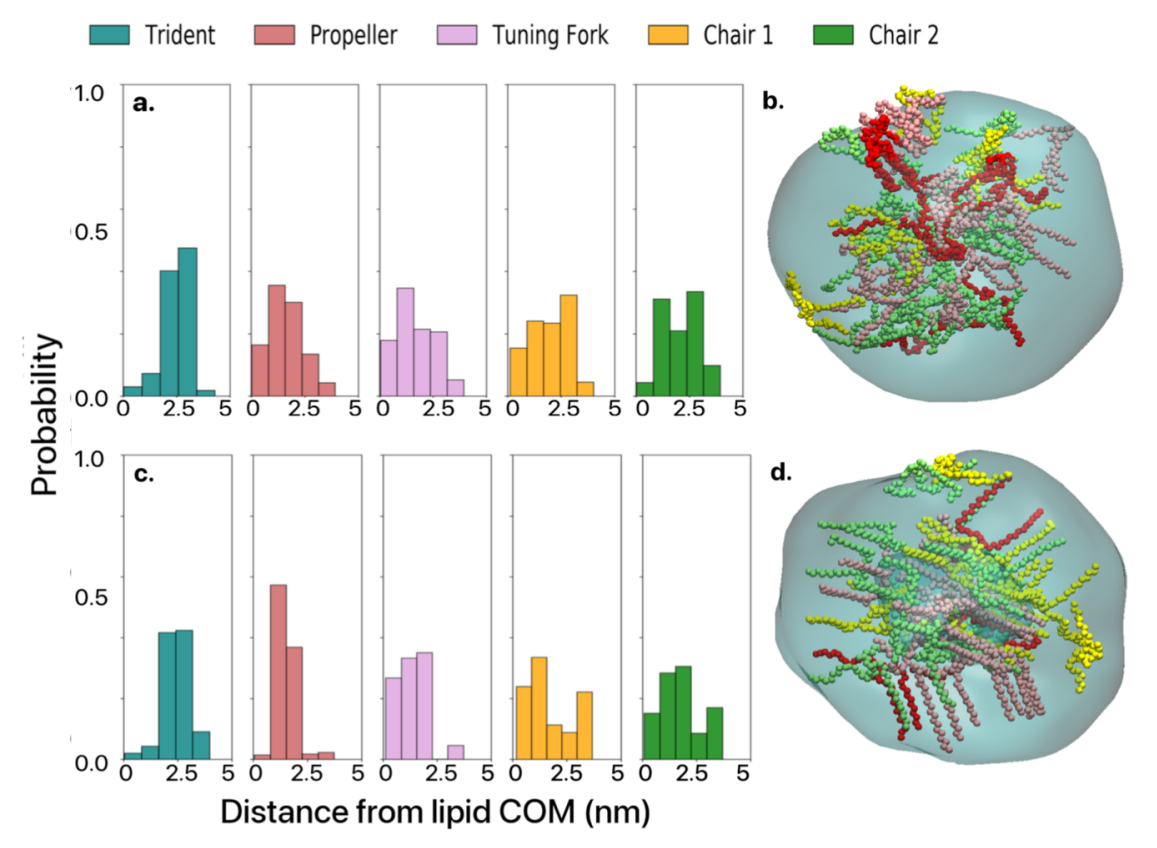

Figure 4: Distributions of the different lipid conformations as a function of distance from the COM of the (a) LA and (c) SA. The percentages of each conformation can be found in Table 3. Figures (b) and (d) show the position of non-trident lipids in the nanoparticle. The location of the trident lipids in the nanoparticle are indicated in transparent cyan.

closer to the COM of the SA nanoparticle than the LA and the range of distances over which the lipids are distributed is larger for the LA. This results from the LA having less crystalline structure than the SA, which is reflected in the slightly larger radius of gyration of the LA $(3.43 \pm 0.01 \mathrm{~nm})$ than the SA $(3.31 \pm$ $0.01 \mathrm{~nm})$.

The non-trident lipids within the aggregates primarily exist in the center of the LA and SA. In the SA, these non-trident lipids also crystallize within the nanoparticle core. The non-trident lipids crystallize with each other, and also with the lipids in the trident conformation, by burying a number of their hydrocarbon tails amongst the hydrocarbon tails of the trident lipids. Although the crystalline non-trident lipids in the core of the SA appear to be less ordered than that of the trident lipids, they are more ordered than the non-trident lipids in the LA.

\section{Lipid conformational transitions}

The probability that a lipid molecule will transition from one conformation to another under these simulation conditions is shown in Table 4. Trident is the most stable conformation as the probability of lipids being in the trident conformation is the highest. Also, when a lipid molecule is in a non-trident conformation, it is most likely to transition to the trident conformation. These two findings are consistent with the understanding that the trident conformation is the most favorable conformation of triglycerides at the interface with an aqueous environment.

The second most stable conformation in the LA and SA is the tuning fork. However, the probability of transitioning to the tuning fork conformation from any other conformation is the lowest for both the LA and SA as well. The probabilities of transition from one conformation to another is always larger in the LA than in the SA but the rank order of transitions is consistent between the LA and SA. 


\section{Solid Lipid Nanoparticle (SLN)}

\section{Experimental results}

The partial ternary phase diagram for SLNs prepared from tripalmitin (TPN) of 99\% purity (99hTPN) and $\mathrm{C}_{18: 1} \mathrm{E}_{10}$ (Brij O10) in $\mathrm{H}_{2} \mathrm{O}$ upon storage at ambient conditions for a month shows three regions of interest (see SI). Specifically, the SLNs within the three regions differ in appearance as the concentration of TPN increases, ranging from clear SLNs in Region A, through to bluish/translucent samples in Region B, and cloudy/milky SLNs in Region C. The SLN stock selected for the SANS studies, namely those comprising of $8 \mathrm{wt} \% \mathrm{TPN}$ and $20 \mathrm{wt} \% \mathrm{C}_{18: 1} \mathrm{E}_{10}$, was from Region C. Significantly, for the present SANS studies, no difference in the extent of SLN existence was seen when the $\mathrm{H}_{2} \mathrm{O}$ was replaced with an equivalent volume of $\mathrm{D}_{2} \mathrm{O}$ suggesting that the isotopic replacement had no significant effect on the SLNs.

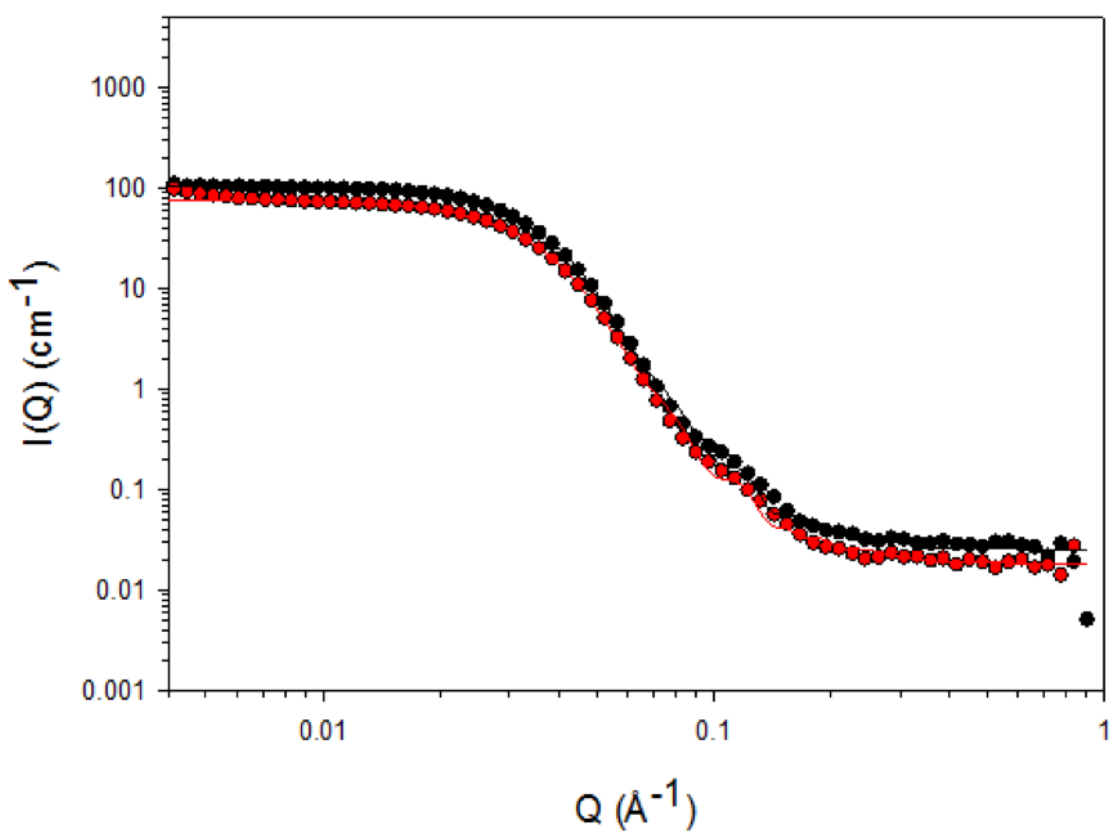

Figure 5: The variation in the neutron scattering intensity as a function of the scattering vector (Q) for TPN-containing SLNs of composition of $0.8 \% \mathrm{w} / \mathrm{w}$ tripalmitin (TPN) and $2.0 \% \mathrm{w} / \mathrm{w} \mathrm{C}_{18: 1} \mathrm{E}_{10}$ as a function of TPN purity (black circles are $85 \mathrm{~h} \mathrm{TPN}$ and red circles are $99 \mathrm{~h}$ TPN) at $2980.1 \mathrm{~K}$. The best fit to the SANS data (solid line) obtained using a core-shell model for the form factor and a hard sphere structure factor to account for the interparticulate interactions.

Figure 5 shows the variation in the total measured scattering with the scattering vector (Q) for the SLNs diluted to a final concentration of $0.8 \mathrm{wt} \% \mathrm{TPN}$ and $2 \mathrm{wt} \% \mathrm{C}_{18: 1} \mathrm{E}_{10}$ in $\mathrm{D}_{2} \mathrm{O}$. Analysis of the SANS data showed that the SLNs were not spherical, but instead were oblate, as the major and minor axis are different lengths (see SI), as has been previously reported from SANS data of steric acid and Tween20 SLNs [40]. In the context of the present study, spherical SLN are generally associated with the $\alpha$-form polymorph whilst the $\beta$-form typically corresponds to platelet shaped SLN. Therefore the TPN contained in the SLN have undergone a polymorphic transition and are no longer in the less stable $\alpha$-form. To probe this observation further, the effect of using TPN of a lower purity on both the phase behavior and properties of the resulting SLNs, as assessed by SANS (see SI), was examined. Whilst the purity of TPN had no influence on the 
composition range over which SLNs are found in Region C, the TPN purity had an effect on the extent of Regions A and B, which were both larger in the case of SLNs containing TPN of $85 \%$ purity. This observation is most likely due to the fact that when TPN of $85 \%$ purity was used, the increased presence of contaminants made the triglyceride less likely to crystalize. This effect is enhanced in the SLNs formed at low TPN concentrations, which result in less cloudy solutions due to the lower refractive index of the TPN. Furthermore, there were very slight differences in the size of the SLNs containing the two different purities TPN as measured by SANS (Table 5) with the SLNs containing 85hTPN being very slightly smaller.

Further analysis of the SANS data (summarised in Table 5) revealed an aggregation number of 659 surfactant molecules per nanoparticle for the lower purity sample. As a result, 650 surfactant molecules were used for the simulation with 229 tripalmitin molecules, to maintain the $2.5 \%$ ratio of surfactant:lipid used in the SANS experiment.

\section{Distribution of lipid conformations}

As shown in Table 3 the trident conformation is adopted by a majority of the lipids. Despite this, the distribution of the lipid conformations shown in Fig. 6 differs from that found in the LA and SA. Whilst the trident conformation has the same symmetrical distribution as in the SLN, there are few non-trident lipids occupying the surface of the nanoparticle in the SLN. Whereas there are lipids in non-trident conformations regularly occupying the surface of the SA and LA. This difference is driven by the stabilization of the surface lipids by the surfactant molecules. Since the surfactant now occupies the surface of the nanoparticle, it shields the hydrophobic lipid tails from exposure to water. The presence of surfactant at the surface also makes it a sterically unfavorable location for non-trident lipids.

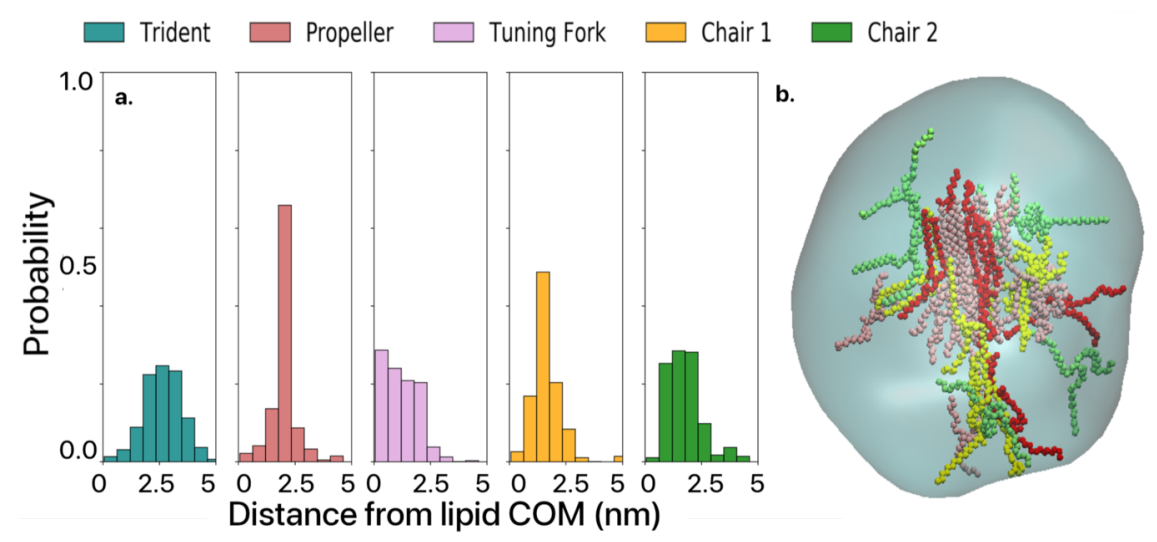

Figure 6: (a) Distribution of different lipid conformations throughout the SLN relative to the COM of the nanoparticle. (b) A representative snapshot of the SLN which shows the position of the non-trident lipids.

Analysis of the lipid distribution suggests that non-trident lipids, which were at the surface in the LA and SA aggregates, instead occupy the core of the SLN. This is reflected by sharper peaks at lower distances in the histograms of the non-trident conformations. These trends suggest that the shell of crystalline trident lipids remains intact.

When visualizing the location of the lipid molecules with non-trident conformations, it is clear that they also crystallize in the core, as was seen in the SA. The fact that very little water penetrates into the core of the nanoparticle allows the lipid molecules to adopt non-trident conformations and remain stable enough to crystallize. Closer analysis of these non-trident lipids reveals that the different conformations attempt to crystallize with each other. This results in the formation of a crystalline solid with considerable defects. If drugs or other small bioactive molecules are localized amongst the lipids then this is likely where they are encapsulated. 


\section{Lipid conformational transitions}

When comparing the transitions between different lipid conformations in the SLN to those in the SA, there are two key differences (Table 4). Firstly, the lipids in the trident conformation are more likely to transition to a non-trident conformation in the SLN than in the SA. In particular, there is a relatively high probability for lipids in a trident conformation to transition to the chair conformation in the SLN. The second significant difference is that the tuning fork conformation is significantly more stable in the SLN than in SA, as inferred by the relative probabilities of lipids in a tuning fork conformation that remain in that conformation. Both of these are consistent with having a more ordered crystalline core in the SLN than in the SA (shown in RDFs in the SI). Therefore, it appears that the surfactants in the SLN stabilize the crystalline core.

\section{Surfactant distribution}

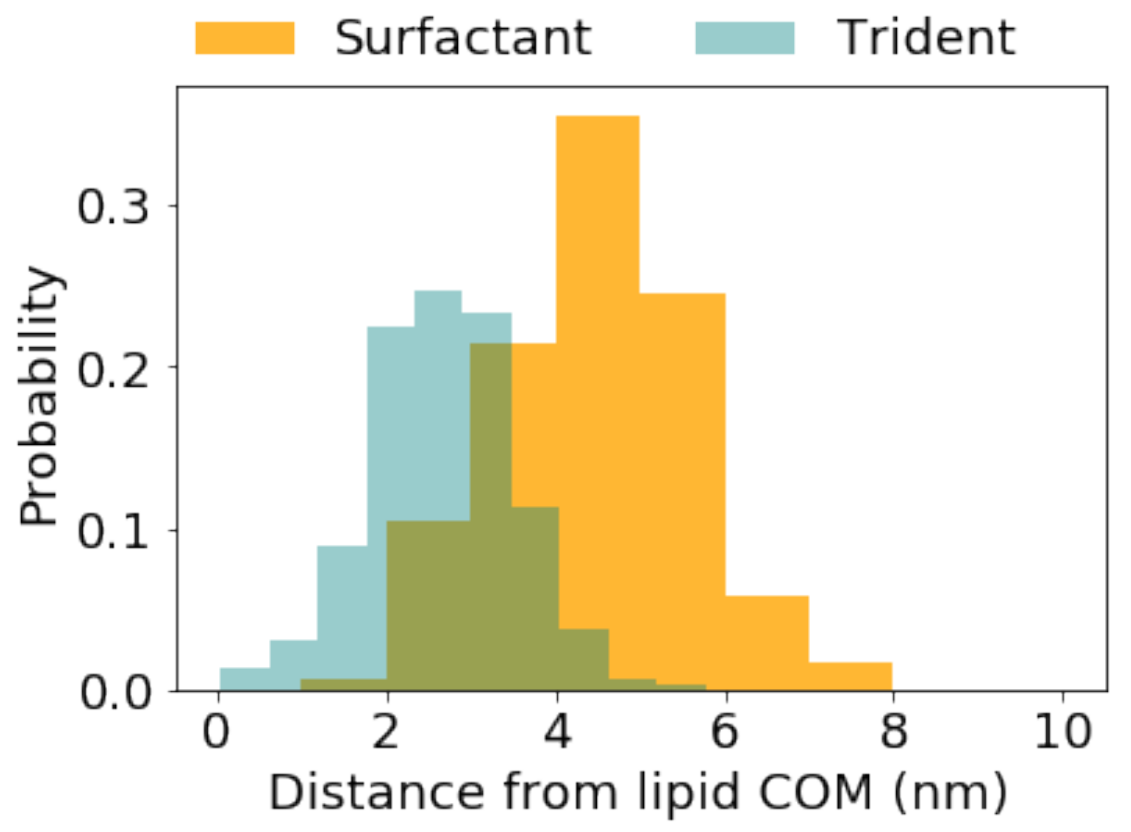

Figure 7: Distribution of the trident lipids and the surfactant from the COM of the lipid core.

The penetration of surfactant into the lipids has been quantified by examining the distribution of the surfactant as a function of distance from the lipids' COM (Fig. 7). The surfactant molecules do not penetrate to the center of the lipid core; the mean minimum distance between the surfactant molecules and the lipid aggregate $\mathrm{COM}$ is $4.5 \mathrm{~nm}$. By means of comparison, the trident lipids sit at a mean distance of $\sim 2.7$ $\mathrm{nm}$ from the lipid COM. The surfactants do not lie exclusively on the surface. Instead, the hydrocarbon chains of some surfactant molecules bury into the interfaces between the different regions of the crystalline trident lipids (Fig. 8a). Figures 8b and 8c illustrate how the the surfactant molecules settle into the spaces between these different crystalline regions and form an approximately spherical nanoparticle, as indicated by its eccentricity (Table 2). In doing so the surfactant molecules shield their hydrocarbon tails, as well as those of the lipid molecules at the edges of these crystalline regions, from the aqueous environment.

It is clear that the surfactant exists predominantly at the surface of the SLN. However, the SASA values of the ester oxygens in the SLN lipid molecules (see SI) show that the ester groups remain exposed to water, albeit to a lesser extent than those in the SA. Therefore, since the surfactant exists predominantly at the interfaces between different crystalline regions, the tripalmitin ester groups are still able to interact directly with the aqueous environment. 


\section{Surfactant morphology}
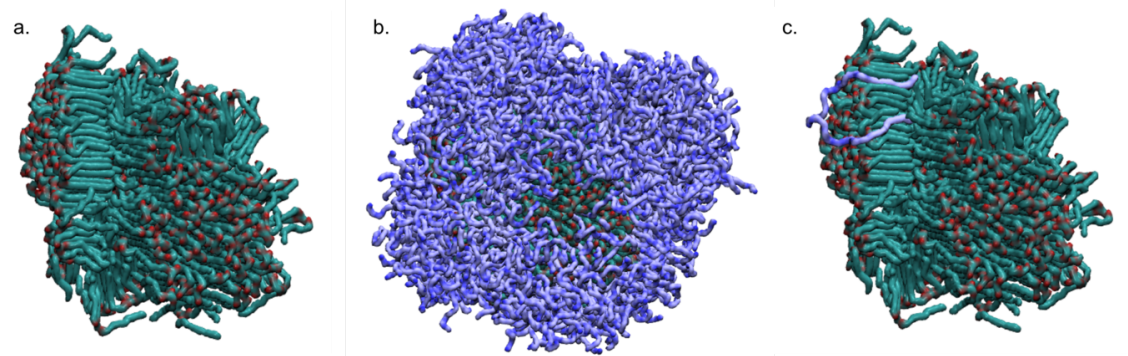

Figure 8: Snapshots of the (a) lipid core of the SLN; (b) lipid and surfactant molecules of the SLN; and (c) two surfactant molecules that have buried their tails within a crystalline region consisting of trident lipid molecules. Note that the lipid oxygens shown in red, the lipid carbons in blue, the surfactant carbons in lilac and the surfactant oxygens in purple.

The conformation of each of the surfactant molecules within the SLN is driven by interactions with the aqueous environment. A detailed understanding of the surfactant conformation within the SLN can be obtained from the SASA values of each carbon atom in the surfactant chains (see SI). Since the SASA values continue to increase along the carbon chain of poly(ethylene glycol), the surfactant molecules adopt a conformation that maximize the preferred interactions of the hydrocarbons and the poly(ethylene glycol) chain. The hydrocarbon tails are either buried amongst the trident lipid molecules or they interact with the hydrocarbon chains of other surfactants at the surface of the SLN (see surfactant contact maps in SI). Meanwhile, the poly(ethylene glycol) groups are immersed in aqueous solution.

Additionally we can ascertain that the hydrocarbon chain is in a more extended conformation than the considerably more flexible polyethylene glycol chain. The hydrocarbon tail $(\mathrm{C} 1$ - C18) was found to have an average end-to-end length approximately $82 \%$ of its theoretical maximum length, whereas the polyethylene glycol chain (C18 - C38) has an average end-to-end length that is $42 \%$ of its maximum length. Combining these results with those obtained by visual inspection of the trajectories, we confirm that the poly(ethylene glycol) portion of the surfactant forms highly hydrated coils emanating from the surface of the SLN.

\section{Discussion}

In this manuscript, we have used a combination of experimental methods including small-angle neutron scattering and a series of all-atom MD simulations to investigate the detailed internal structure of a liquid lipid aggregate, solid lipid aggregate and solid lipid nanoparticle. We first experimentally identified the ratio of $\mathrm{C}_{18: 1} \mathrm{E}_{10}$ surfactants to tripalmitin triglycerides which result in a stable solid lipid nanoparticle. We then used SANS to determine the aggregation numbers of surfactants and lipids in the SLNs. These aggregation numbers were then used as a basis to build our simulated systems. We have successfully used a similar approach for surfactant micelles in the past ${ }^{[41],[42]}$. These simulations were used to provide the first atomistic level description of each of the three different nanoparticles. In doing so, we provide a description of the molecular conformations of tripalmitin triglycerides in each system. Also, we have shown how these triglycerides go through conformational transitions in the different systems. In addition, we have demonstrated how the $\mathrm{C}_{18: 1} \mathrm{E}_{10}$ surfactants stabilize the crystalline facets of the tripalmitin core of a SLN by shielding the hydrophobic sections of the lipids from water.

Although the existence of $\alpha$-form crystalline tripalmitin SLN has been confirmed experimentally ${ }^{[18]}$, there is no clear polymorph present within any of our simulations. The lack of a pronounced crystalline morphology for the SLN in the present study could be the result of using a surfactant that interacts with the lipids in a 
different way, and therefore alters the rate of polymorphic transitions ${ }^{[15]}$. Additionally, the small size of the simulated nanoparticle may result in no clear polymorph being observed ${ }^{[43]}$. The SLN in this simulation has an overall $R_{g}$ of $5.53 \mathrm{~nm}$ and the $R_{g}$ of the SA is smaller at $3.3 \mathrm{~nm}$. The tripalmitin SLN that was found to have an $\alpha$-crystalline structure had a particle size in excess of $100 \mathrm{~nm}$. Whilst these values are not directly comparable they give an indication as to the different scales of the SLN. A significantly larger SLN would have an increased bulk lipid area, where the lipids are not exposed to water, and are therefore able to adopt the non-trident conformations seen in these simulations. The SLN would then have the chance to develop into one of the crystalline polymorphs. Experimental and computational studies of bulk triglycerides have shown that the tuning fork and chair conformations are the most common in bulk lipid, which is also reflected in our SLN lipid core ${ }^{[20],[23],[24],[16]}$. Additional simulation work confirms the preference of lipids to adopt the trident conformation at the lipid/water interface ${ }^{[44],[24]}$ and experimental evidence has shown that lipids at the air/water interface are also in the trident conformation ${ }^{[45]}$.

The size of the SLN which we have simulated is in good agreement with the measured size of the SLN as found from SANS. The difference in $R_{g}$ between the SA and the SLN is $2.22 \mathrm{~nm}$. This is almost identical to the shell thickness of $2.3 \mathrm{~nm}$ (Table 5) calculated from the SANS data. Figure 7 shows significant amounts of surfactant located $6-8 \mathrm{~nm}$ from the lipid COM which is also in close agreement with the SLN sizes recorded from the SANS experiments. The larger SANS radius is reflective of the different parameters measured. The simulations record the radius of gyration but the SANS data documents the sphere radius and incorporates the hydrating water and so would be slightly larger than the $R_{g}$, as is seen here.

In the SLN, unlike in the SA, the non-trident lipids in the lipid core of the nanoparticle do not bury their triglyceride tails into the shell of crystalline trident lipids. Instead, the non-trident lipids crystallize primarily with each other in the lipid core of the SLN. Additionally, the mean minimum distance from trident lipids to the COM of the lipid core is $2.36 \pm 0.62 \mathrm{~nm}$ in the SA, whilst for the SLN it is $2.67 \pm 0.85 \mathrm{~nm}$. Therefore, the nanoparticle core is expanding to better accommodate the non-trident lipids in its core as they crystallize. Expansion of the nanoparticle core is caused by surfactant molecules at the edges of the crystalline regions of trident lipids, at the surface of the SLN, these surfactant molecules shield the hydrocarbon tails of the lipids at the edge of these regions from the aqueous environments. The surfactants replace the non-trident lipids that served the same purpose in the SA, and therefore allow those lipids to move into the lipid core of the nanoparticle, taking up crystalline conformations. The enhanced crystalline lipid content of the SLN as compared to the $\mathrm{SA}$ is demonstrated by the RDF between $\mathrm{C} 18$ atoms in the lipid molecules, which shows larger peaks at smaller distances for SLN than for the SA (see SI). The lipid molecules pack closer together in both the core and the trident crystalline regions of the SLN than in the SA. The stronger van der Waals forces between the trident tails, caused by their increased extension and closer proximity, make it less favorable for the non-trident tails to penetrate the sphere, encouraging the lipids to crystallize with each other instead.

\section{Conclusions}

The results of MD simulations presented in this manuscript allow us to determine the atomistic structure of the liquid and solid lipid aggregates consisting of tripalmitin triglycerides, and of solid lipid nanoparticles consisting of tripalmitin and $\mathrm{C}_{18: 1} \mathrm{E}_{10}$ surfactants. The liquid lipid aggregate consists of a lipid shell at the interface with its aqueous environment, in which the lipids primarily are found in a trident conformation. The trident conformation allows the triglycerides to maximise the contact between their polar ester groups and surrounding water molecules and the hydrophobic interaction between their hydrocarbon tails. In the core of the liquid aggregate, we found that the lipids are liquid and consist of a mixture of different non-trident lipid conformations. The solid lipid aggregate also has a lipid shell at the interface with the surrounding water. However, the lipids in this outer shell of the solid aggregate form various crystalline facets. These crystalline facets seem to be stablised by the interdigitation of the tails of the triglycerides in the interfacial shell of the solid aggregate with tails of triglycerides in the core. We didn't observe any such crystalline facets in the liquid aggregate. We believe this is due to the increased amount of non-trident lipid molecules 
in the outer shell of the liquid aggregate and the reduced interaction between the lipids in the liquid core and the lipids in the outer shell when compared to that in the solid aggregate.

In the solid lipid nanoparticle with a composition taken from our SANS experiments, we again observed a crystalline lipid shell at the interface with the surrounding water, which was made up of entirely of lipids in the trident conformation. The core of the solid lipid nanoparticles is found to consist of primarily non-trident lipid conformations which crystallise with each other. As in the solid aggregate, we observe that the lipids in the outer shell form different crystalline facets that are even more ordered than in the solid aggregate. The $\mathrm{C}_{16: 1} \mathrm{E}_{10}$ surfactants interact more strongly with one another than with the lipid molecules, and as a result cluster in the space between the different facets in the outer lipid core. In doing so, the hydrocarbon tails of the surfactants are shielded from their aqueous environment, and also shield the hydrocarbon tails of the lipids on the edges of the various facets of the outer shell. We found that the poly(ethylene glycol) portion of the surfactant forms highly hydrated coils that emanate from the surface of the solid lipid nanoparticle. While the general behaviour of the surfactants is as would be expected, we have shown direct evidence of the mechanisms that lead to their behaviour using the results of our MD simulations.

The findings presented in this work provide foundations to better understand the capabilities of SLNs to carry and deliver bioactive molecules. By understanding in atomistic detail, the molecular structure and morphology of these nanoparticles in the liquid and solid state, and also when the solid nanoparticles are stabilized by surfactants, one can better understand the available space within the nanoparticles to carry small molecules. Additionally, it can be seen how the interior of these nanoparticles change under different conditions. For example, in the use of SLN as drug delivery vehicles, the precise location(s) of drug molecules after solubilization is unknown. The crystalline arrangement of the trident shell would make it an unfavorable location for drug localization, however, this study has revealed the presence of a mixed-lipid core with large defects within its crystalline packing. This suggests that if drug molecules are encapsulated within the SLN, they must be located within this mixed-lipid core where there are voids in the crystalline packing. Mazuryk et al. used Fourier transformed infrared spectra (FT-IR) and small-angle neutron scattering (SANS) to show that the drug rapamycin incorporated between the fatty acid chains and caused a significant drop in the melting point of the excipient, which led to a distortion of the internal structure of the SLN ${ }^{[46]}$. Additionally, drug molecules could localize throughout the surfactant at the surface. In this work, we have shown that the surfactants are more flexible than the crystalline lipids. If a hydrophobic drug molecule localizes within the surfactant layer then previous work suggests it would be located amongst the dehydrated hydrocarbon chains of the surfactant ${ }^{[47]}$.

\section{Acknowledgements}

Via our membership of the UK's HEC Materials Chemistry Consortium, which is funded by EPSRC (EP/L000202), this work used the ARCHER UK National Supercomputing Service (http://www.archer.ac.uk) and the UK Materials and Molecular Modelling Hub (MMM Hub) for computational resources, which is partially funded by EPSRC (EP/P020194/1) to carry out the MD simulations reported in this manuscript. D.L.P. and C.D.L. acknowledge the support by the Biotechnology and Biological Sciences Research Council (BB/M009513/1) via the London Interdisciplinary Doctoral Programme (LIDo). Additionally, R.M.Z. and C.D.L. acknowledge the research environment provided by the EPSRC Centre for Doctoral Training in Cross-Disciplinary Approaches to Non-Equilibrium Systems (CANES, EP/L015854/1). This work was supported by the STFC though the award of neutron beam time. Also, we acknowledge the Royal Thai Government for awarding a scholarship to L.O. and the National Nanoteachnology Centre (Nanotec) for awarding L.O. leave of absence for her studies. 


\begin{tabular}{c||c|c|c|c|} 
Initial/Final & $\mathbf{T}$ & $\mathbf{P}$ & $\mathbf{T F}$ & $\mathbf{C}$ \\
\hline \hline \multirow{3}{*}{$\mathbf{T}$} & 95.4 & 1.0 & 1.3 & 2.3 \\
& 98.7 & 0.5 & 0.4 & 0.4 \\
$\mathbf{3}$ & 89.8 & 1.9 & 1.3 & 7.0 \\
\hline $\mathbf{P}$ & 13.3 & 73.8 & 5.0 & 7.9 \\
& 6.3 & 86.8 & 1.8 & 5.1 \\
$\mathbf{3}$ & 8.9 & 83.0 & 3.4 & 4.6 \\
\hline \multirow{2}{*}{$\mathbf{T F}$} & 16.1 & 5.2 & 76.1 & 2.7 \\
& 7.1 & 2.2 & 90.7 & 0.0 \\
& 1.9 & 1.2 & 96.8 & 0.0 \\
\hline \multirow{2}{*}{$\mathbf{C}$} & 19.5 & 5.7 & 1.9 & 73.0 \\
& 6.1 & 4.5 & 0.0 & 89.4 \\
\hline \hline
\end{tabular}

Table 4: Transition probabilities in \% over the final 5 ns of the trajectory between 10 ps snapshots in the MD simulations for the LA (red), SA (blue) and SLN (green). Trident (T), Propeller (P), Tuning Fork (TF) and Chair $(\mathrm{C})$

\begin{tabular}{l||l|l|l|l|l|l} 
Sample & Region & $\begin{array}{l}\text { Shell Thick- } \\
\text { ness (nm) }\end{array}$ & $\begin{array}{l}\text { Sphere } \\
\text { radius } \\
(\mathbf{n m})\end{array}$ & $\begin{array}{l}\text { Hydration } \\
\mathbf{( \% )}\end{array}$ & $\mathbf{N}_{\text {agg }}$ & $\begin{array}{l}\text { Surface area of } \\
\text { surfactant }\left(\AA^{\mathbf{2}}\right)\end{array}$ \\
\hline \hline $\mathbf{8 5 \%}$ TPN & B & 2.3 & 9.1 & 20 & 659 & 78 \\
\hline $\mathbf{9 9 \%}$ TPN & C & 2.3 & 8.5 & 20 & 671 & 78
\end{tabular}

Table 5: Parameters used to obtain the best fits to the SANS data for TPN-containing SLNs of composition of $0.8 \%$ wt TPN/2 \%wt $\mathrm{C}_{18: 1} \mathrm{E}_{10}$ (Brij O10) as a function of TPN purity using a core-shell ellipsoid model for the form factor together with a hard sphere structure to account for interparticulate interactions at 298 $\pm 0.1 \mathrm{~K}$. 


\section{References}

[1]M. R. Gasco, S. Morel, Il Farmaco 1990, 45, 1127.

[2]B. Siekmann, K. Westesen, Pharm. Pharmacol. Lett. 1992, 1, 123.

[3]R. H. Muller, W. Mehnert, J.-S. Lucks, C. Schwarz, A. zur M uhlen, H. Weyhers, C. Freitas, D. R uhl, Eur. J. Pharm. Biopharm. 1995, 41, 62.

[4]H. Bunjes, J. Pharm. Pharmacol. 2010, 62, 1637.

[5]H. Bunjes, Curr. Opin. Colloid Interface Sci. 2011, 16, 405.

[6]J.-H. Kim, Y. Kim, K. H. Bae, T. G. Park, J. H. Lee, K. Park, Mol. Pharm. 2015, 12, 1230.

[7]R. H. Muller, K. Mäder, S. Gohla, Eur. J. Pharm. Biopharm. 2000, 50, 161.

[8]W. Mehnert, K. M ader, Adv. Drug Deliv. Rev. 2001, 47, 165.

[9]K. Westesen, H. Bunjes, Int. J. Pharm. 1995, 115, 129.

[10]K. Westesen, B. Siekmann, Int. J. Pharm. 1997, 151, 35.

[11]R. H. Muller, M. Radtke, S. A. Wissing, Adv. Drug Deliv. Rev. 2002, 54, 131.

[12]D. J. McClements, E. A. Decker, J. Weiss, J. Food Sci. 2007, 72, 109.

[13]D. J. McClements, Y. Li, Adv. Colloid Interface Sci. 2010, 159, 213.

[14]K. Westesen, B. Siekmann, M. H. J. Koch, Int. J. Pharm. 1993, 93, 189.

[15]H. Bunjes, M. H. J. Koch, K. Westesen, J. Pharm. Sci. 2003, 92, 1509.

[16]C. Himawan, V. M. Starov, A. G. F. Stapley, Adv. Colloid Interface Sci. 2006, 122, 3.

[17]S. Das, A. Chaudhury, AAPS PharmSciTech 2010, 12, 62.

[18]H. Bunjes, F. Steiniger, W. Richter, Langmuir 2007, 23, 4005.

[19]G. D. Kalaycioglu, N. Aydogan, Colloids Surf. A 2016, 510, 77.

[20]A. Hall, J. Repakova, I. Vattulainen, J. Phys. Chem. B 2008, 112, 13772.

[21]A. Brasiello, S. Crescitelli, G. Milano, Faraday Discuss. 2012, 158, 479.

[22]C. J. MacDougall, M. S. Razul, E. Papp-Szabo, F. Peyronel, C. B. Hanna, A. G. Marangoni, D. A. Pink, Faraday Discuss. 2012, 158, 425.

[23]A. Pizzirusso, A. Brasiello, A. D. Nicola, A. G. Marangoni, G. Milano, J. Phys. D 2015, 48, 494004.

[24]A. S. Tascini, M. G. Noro, R. Chen, J. M. Seddon, F. Bresme, Phys. Chem. Chem. Phys. 2018, 20, 1848.

[25]X. F. Fernandez-Luengo, J. Camacho, J. Faraudo, Nanomaterials 2017, 7, 461.

[26]A. S. Tascini, M. G. Noro, J. M. Seddon, R. Chen, F. Bresme, Phys. Chem. Chem. Phys. 2019, 21, 1471.

[27]A. Pizzirusso, F. Peyronel, E. D. Co, A. G. Marangoni, G. Milano, Journal of the American Chemical Society 2018, 140, 12405.

[28]R. M. Hathout, A. A. Metwally, Eur. J. Pharm. Biopharm. 2016, 108, 262.

[29]P. Wasutrasawat, H. Al-Obaidi, S. Gaisford, M. J. Lawrence, W. Warisnoicharoen, Eur. J. Pharm. Biopharm. 2013, 85, 365. 
[30]P. Wasutrasawat, Formation and Physicochemical Properties of Non-Ionic Oil-in-Water Nanoemulsions Containing Liquid and Solid Triglycerides, PhD thesis, Chulalongkorn University, 2012.

[31].

[32]M. D. Hanwell, D. E. Curtis, D. C. Lonie, T. Vandermeerschd, E. Zurek, G. R. Hutchison, J. Cheminformatics 2012, 4, DOI 10.1186/1758-2946-4-17.

[33]J. B. Klauda, R. M. Venable, J. A. Freites, J. W. O'Connor, D. J. Tobias, C. Mondragon-Ramirez, I. Vorobyov, A. D. MacKerell, R. W. Pastor, J. Phys. Chem. B 2010, 114, 7830.

[34]W. E. Reiher, Theoretical Studies of Hydrogen Bonding, PhD thesis, Harvard University, 1985.

[35]B. Hess, C. Kutzner, D. Van Der Spoel, E. Lindahl, J. Chem. Theory Comput. 2008, 4, 435.

[36]E. Lindahl, B. Hess, D. van der Spoel, J. Mol. Model. 2001, 7, 306.

[37]D. Van Der Spoel, E. ahl, B. Hess, G. Groenhof, A. E. Mark, H. J. C. Berendsen, Journal of Computational Chemistry 2005, 26, 1701.

[38]M. J. Abraham, T. Murtola, R. Schulz, S. Páll, J. C. Smith, B. Hess, E. Lindahl, SoftwareX 2015, 1-2, 19.

[39]W. Humphrey, A. Dalke, K. Schulten, J. Mol. Graph. 1996, 14, 33.

[40]R. M. Shah, J. P. Mata, G. Bryant, L. de Campo, A. Ife, A. V. Karpe, S. R. Jadhav, D. S. Eldridge, E. A. Palombo, I. H. Harding, Part. Part. Syst. Charact. 2018, 36, 1800359.

[41]D. T. Allen, Y. Saaka, M. J. Lawrence, C. D. Lorenz, J. Phys. Chem. B 2014, 118, 13192.

[42]C. D. Lorenz, C.-M. Hsieh, C. A. Dreiss, M. J. Lawrence, Langmuir 2011, $27,546$.

[43]H. Bunjes, M. H. J. Koch, K. Westesen, Langmuir 2000, 16, 5234.

[44]A. Bacle, R. Gautier, C. L. Jackson, P. F. J. Fuchs, S. Vanni, Biophysical Journal 2017, 112, 1417.

[45]M.-C. Michalski, P. Brogueira, A. G. da Silva, B. Saramago, European Journal of Lipid Science and Technology 2001, 103, 677.

[46]J. Mazuryk, T. Deptuła, A. Polchi, J. Gapiński, S. Giovagnoli, A. Magini, C. Emiliani, J. Kohlbrecher, A. Patkowski, Colloids Surf. A 2016, 502, 54.

[47]D. Attwood, P. H. Elworthy, M. J. Lawrence, J. Pharm. Pharmacol. 1989, 41, 585. 